\title{
Journal of Diabetes Research and Therapy
}

\section{Recommendations for the Use of Statins in Patients with Diabetes Mellitus or Prediabetes. Conclusions of a Consensus Group}

\section{Millán $\mathrm{J}^{1^{\star}}$, Ascaso $\mathrm{JF}^{2}$, Barrios $\mathrm{V}^{3}$, Cases $\mathrm{A}^{4}$, Serrano $\mathrm{A}^{5}$, Pascual $\mathrm{V}^{6}$, Pedro-Botet $\mathrm{J}^{7}$ and Pintó $X^{8}$}

IInternal Medicine Service, Gregorio Marañón General University Hospital, Complutense University of Madrid, Madrid, Spain

${ }^{2}$ Endocrinology Service, Hospital Clínico, Faculty of Medicine, Valencia, Spain

${ }^{3}$ Cardiology Service, Ramón y Cajal University Hospital, University of Alcalá, Madrid, Spain

${ }^{4}$ Nephrology Service, Hospital Clinic, University of Barcelona, Barcelona, Spain

${ }^{5}$ Family Medicine Service, Repelega Health Centre, Portugalete, Bizkaia, Spain

${ }^{6}$ Palleter Health Centre, Castellón, Spain

${ }^{7}$ Lipids Unit, Endocrinology Service, Hospital del Mar, Autonomous University of Barcelona, Barcelona, Spain ${ }^{8}$ Lipids Unit, Internal Medicine Service, University Hospital of Bellvitge, Barcelona, Spain

*Corresponding author: Jesus Millán, Internal Medicine Service, Gregorio Marañón General University Hospital, Complutense University of Madrid, Madrid, Spain, E-mail: jesus.millan@salud.madrid.org
Received date: 18 Apr 2017; Accepted date: 12 Jul 2017; Published date: 17 Jul 2017.

Citation: Millán J, Ascaso JF, Barrios V, Cases A, Serrano A, et al. (2017) Recommendations for the Use of Statins in Patients with Diabetes Mellitus or Prediabetes. Conclusions of a Consensus Group. J Dia Res Ther 3(2): doi http://dx.doi.org/10.16966/23805544.128

Copyright: ( 2017 Millán J, et al. This is an open access article distributed under the terms of the Creative Commons Attribution License, which permits unrestricted use, distribution, and reproduction in any medium, provided the original author and source are credited.

\begin{abstract}
The contribution of statins in the treatment of cardiovascular risk of lipid origin has been a determining factor in the reduction of morbimortality from cardiovascular diseases due to a decrease in risk. However, statins are not exempt from possible adverse effects. One of them is their capacity to cause disorders in the carbohydrate metabolism, increasing the risk of new-onset diabetes in patients treated with statins significantly, although the cardiovascular benefit of their use is always higher. Yet, especially in diabetic or pre-diabetic patients, the statin of choice may condition the safety in this area, given that the diabetogenic profile of the different molecules differ.

In particular, the objectives of this study were to summarize the expert's opinion on whether all statins have a similar or different diabetogenic effect and to establish recommendations on the statin selection based on the patient characteristics.

In order to ascertain the opinion of the experts (primary care physicians and other specialists with experience in the management of this type of patient) we conducted a Delphi study to evaluate the consensus rate on diverse aspects related to the diabetogenicity of different statins, and the factors that influence their choice.

On the basis of the consensus agreements reached among practitioners of different specialities, and considering the availability of greater and better scientific evidence, this work groups together the recommendations to consider when selecting a statin in individuals with prediabetes or diagnosed diabetes. Such recommendations are grouped in relation with the patient, each individual's own carbohydrate metabolism, and the specific characteristics of every statin. These recommendations could facilitate the rational use of one statin or another in order diminish the diabetogenic potential shown by some of them.
\end{abstract}

Keywords: Diabetes; Prediabetes; Statins; Selection criteria

\section{Introduction}

In spite of the clinical benefit of statins in cardiovascular prevention, it is necessary to point out their possible adverse effects. In this sense, it is important to mention that their diabetic effects may mitigate their benefits, even though the cardiovascular benefit will always outweigh the risk resulting from the appearance of diabetes. Therefore, any statin with sufficient hypocholesterolemic capacity to achieve the objective of lipid control, and with neutral or beneficial effects on the carbohydrate metabolism and on the risk of developing diabetes, should be of choice, above all in patients with real or potential alterations in glucose metabolism.

Thanks to randomised clinical studies, post-hoc studies, largescale meta-analyses and Mendelian randomisation methods, it has been possible to confirm that statins may increase the risk of diabetes, especially in those patients with a metabolic predisposition to suffer from it. The intensity of the statin, its dose and the duration of the treatment may be other determining factors in such effects (Table 1), and the mechanisms may be diverse (Table 2). However, some of the statins have shown to be safer in this field: this is the case of pravastatin, although there are discordant results, and pitavastatin, with consistent results across the studies that show favourable data for its use [1].

The impact of this data is that the choice of a specific statin in a patient with frank diabetes or at risk of suffering from it, should attend not only to its LDL-reducing capacity, but also to its safety or the associated side-effects on carbohydrate metabolism. In this sense, it is necessary to formulate some questions that should be addressed. Are all the statins equal? What does the choice of the statin depend upon? What are the variables to be considered when selecting a statin? And finally: How does any pre-existence or possible existence of carbohydrate metabolism disorder, whether prediabetes or diagnosed diabetes mellitus, influence this choice?

In fact, the European Atherosclerosis Society (EAS) has established recommendations for the use of statins in patients with a high risk of 
Table 1: Comparative diabetogenic effect between statins (excluding pitavastatin)

\begin{tabular}{|c|l|c|c|}
\hline \multicolumn{1}{c|}{ Quality } & \multicolumn{1}{|c|}{ Statin } & Increase in risk & Comparator \\
\hline \multirow{3}{*}{ Statin } & Rosuvastatin & $+25 \%$ & \\
\cline { 2 - 3 } & Atorvastatin & $+12 \%$ & \multirow{2}{*}{ Pravastatin } \\
\cline { 2 - 3 } & Simvastatin & $+11 \%$ & \\
\hline \multirow{2}{*}{ Dose } & High dose & $+30 \%$ & \multirow{2}{*}{ Low dose } \\
\cline { 2 - 3 } & Moderate dose & $+22 \%$ & \multirow{2}{*}{ Pravastatin } \\
\hline \multirow{2}{*}{ Potency } & High potency & $+22 \%$ & \\
\cline { 2 - 3 } & Moderate potency & $+11 \%$ & \\
\hline
\end{tabular}

Table 2: Most plausible mechanisms of statin diabetogenicity

Blockade of calcium channels in beta cells, with a consequent reduction in insulin secretion

Blockade in the synthesis of intermediate products of intracellular cholesterol metabolism

Reduction in glucose transporter GLUT4, which controls insulin-mediated cellular intake

Reduction of the Coenzyme Q10 levels, with subsequent reduction of GLUT4

Cholesterol overload in beta cells, with possible cytotoxic effect

developing diabetes [2], and suggests the monitorization of baseline glucose and glycosylated haemoglobin levels at the beginning of the treatment and three months later, although there are no referral criteria for selecting drugs.

\section{Development of Diabetes Mellitus in Patients treated with Statins}

Logically, the most consistent evidence comes from the meta-analyses. A first meta-analysis with 5 studies had not shown an increased risk of diabetes [3], but it was previous to the inclusion of the JUPITER study [4] with rosuvastatin, in which a $26 \%$ increase in the risk of appearance of new cases of diabetes was confirmed. Once the studies with rosuvastatin are included, the increase in globally considered risk rose to $13 \%$ [5], although the inclusion of studies with pravastatin concealed this effect, since the WOSCOPS study with this statin had shown a favourable effect on diabetogenic risk [6]. However, it is not unequivocal that pravastatin produces this favourable effect, because in the LIPID study [7] it was neutral and in the PROSPER study the risk was increased by $32 \%$ [8].

A classical evidence is discerned from a global meta-analysis with different statins that included 13 studies with approximately 100,000 participants $[2,9]$ in which a global increase in the risk of diabetes of $9 \%$ at four years was confirmed.

Not all statins display the same risk. A comparative analysis shown a significant increase in the risk of diabetes with rosuvastatin (42\%), atorvastatin (25\%) and simvastatin (14\%) and a non-significant increase with fluvastatin (4\%) and pravastatin (2\%) [10]. These data are consistent with the increase attributed to the different statins when compared with pravastatin [11]: $22 \%$ with atorvastatin, $18 \%$ with rosuvastatin, and $10 \%$ with simvastatin; without increased risk with lovastatin or fluvastatin.

Two further meta-analyses, the first with 5 studies [12] and the second with 17 studies [13] showed that the risk, although variable with different statins, depends on the dose (25\% with $20 \mathrm{mg}$ of rosuvastatin, $15 \%$ with 80 $\mathrm{mg}$ of atorvastatin, and $7 \%$ with $40 \mathrm{mg}$ of pravastatin).

Thus, while rosuvastatin, atorvastatin and simvastatin have shown an increase in risk, pravastatin, lovastatin and fluvastatin generally do not. With respect to pitavastatin, the clearest evidences are in two studies: an individualised meta-analysis of the effects of pitavastatin in 15 studies with more than 1,600 patients without diabetes treated with different doses of pitavastatin, in which no significant variations arose in baseline glycaemia, in the levels of glycosylated haemoglobin, and in the incidence of newonset diabetes [14]; and the most recent results from the J-PREDICT study, in which a decrease of $18 \%$ of the incidence of diabetes occurred when pitavastatin was used in patients with prior glucose intolerance, compared with isolated interventions in lifestyle [15]. Comparatively there are numerous studies showing that pitavastatin is safer than other statins with regard to alterations in glucose metabolism, particularly in relation to atorvastatin, when the method used is direct comparison on the effects on baseline glycaemia, glycosylated haemoglobin values and the incidence of new-onset diabetes [16].

From what has been shown to date by the comparative data and the specific studies on the drug $[14,15]$, it is deduced that pitavastatin is a statin with a medium-high potential regarding LDL reduction, and a neutral or positive effect on glucose metabolism, without any increase (or even with a decrease) on the risk of new-onset diabetes. Therefore, it has been considered as a statin of choice in the treatment of dyslipidaemia in diabetic patients or those at risk of developing diabetes [16].

\section{Methodology to Register Experts' Opinion in Our Medium}

Trying to formulate practical recommendations that may serve as a guide when treating patients with prediabetes or diabetes mellitus, or even at risk of developing it, with statins, a determining factor is the analysis of the scientific evidence available, and also to find out the opinion of experts in the management of these situations, revealing their level of agreement/ disagreement with certain practices.

This would allow us to establish the degree of consensus/discrepancy in the opinion of expert clinical practitioners in the management of patients with dyslipidaemias, regarding the influences in therapeutic decisionmaking, with a special focus on patients with carbohydrate metabolism disorders, and in the selection of a statin according to its capacity to interfere with it.

In prior studies [17-19], it has been described the method used to obtain this opinion from a broad group of experts, consisting on a modified Delphi method.

The modified Delphi method [20] was used to reach the greatest consensus possible from a broad panel of dyslipidaemia experts. It is a structured technique for remote consensus, which maintains its key advantages (controlled interaction, opportunity to reconsider an opinion and statistical validation of consensus) over other technical alternatives and it addresses some of their major drawbacks (opinion biases). Its execution required two successive waves of a structured survey filled out through an online platform. The doctors were able to confidentially compare their personal opinions with the overall opinion of the panel when answering the second wave and could reconsider, where appropriate, their initial criteria on issues where there was no consensus. The study was conducted in four phases: (a) the selection of a scientific committee, responsible for the formulation of the survey questions; (b) the selection of an expert panel of professionals from four specialties (cardiology, endocrinology, internal medicine, nephrology) and family and community medicine with experience in the field of dyslipidaemia; (c) an online survey in two waves and (d) an analysis of results and discussion of conclusions in a meeting held by the scientific committee.

The project's scientific committee revised the contents of the Delphi questionnaire. A bibliographic search prioritising systematic reviews was conducted as well as another critical synthesis of scientific literature through the consultation of bibliographic databases (MEDLINE, EMBASE and the Spanish Medical Index), and a manual review of the references 
obtained to find other references that may be of interest by using keywords such as dyslipidaemia, diabetogenicity or impaired glucose metabolism. Each survey question submitted to the panel for assessment was devised in the form of a positive or negative statement, as a clinical recommendation responding to interesting or controversial aspects in the clinical management of patients with dyslipidaemia and impaired glucose metabolism. The final version of the questionnaire included in this article contained 2 blocks of questions: a) Opinion on the profile of statins in the treatment of dyslipidaemia in patients with impaired glucose metabolism and b) Recommendations for the selection of the lipid-lowering treatment of choice in patients with impaired glucose metabolism.

The experts proposed were selected by the committee based on their medical and scientific expertise and considering their interest in dyslipidaemia. For their recruitment, a "snowball" strategy was used from personal contacts of the committee members, who in turn proposed new candidates from their professional environment [21]. Following this process, 506 professionals were invited of which 497 experts from all autonomous communities in Spain agreed to participate. All of them were practitioner clinical doctors: $58.4 \%$ primary care physicians; $13.7 \%$ endocrinologists; $13.9 \%$ internal medicine; $7.0 \%$ cardiologists; and 7.0\% nephrologists.

A single type of rating scale was proposed for all questions, and the categories were divided into three regions (Disagree; Neutral; Agree), in order to identify the prevalent opinion. All questions had to be answered in wave 1 . In wave 2 , the questions for which consensus was not achieved in wave 1 was asked again. Consensus was deemed to be reached when a question obtained at least $80 \%$ of responses grouped in consensus in disagreement or scores in consensus in agreement.

\section{Multidisciplinary Consensus}

The mission of the consensus was none other than gathering the opinion on clinical practice in the management of dyslipidaemias in patients with carbohydrate metabolism alterations, whether present or potential and thus identifying the best possible practices among the experts. It is also necessary to recognise those elements that enable the correct selection of a statin for these patients. Consequently, the consensus values are none other than those generated by a group of experts with experience in the management of these patients and the use of these drugs, and-ultimately - should allow the definition of a set of recommendations for the selection and use of statins, helping in the decision-making. This is the main goal of the study.

The issues consensuated refer to three fundamental aspects: A) the management of dyslipidaemia in these patients, both from the viewpoint of detection, as the treatment and its monitoring; B) the selection of statins in these patients, as well as the elements that support it; and C) the different diabetogenicity of statins and - in consequence - the diabetogenic profile of the statins available.

Tables 3-5 summarily include the aspects agreed on by consensus in these three aspects. Regarding A) the management of dyslipidaemia: aspects relating to the selection of patients for screening initial therapeutic approach and the parameters to be followed and controlled; B) the factors that condition the selection of a statin both at the time of prescription and during the follow-up and C) the diabetogenic profile of statins, whether it can be considered a class effect or - to the contrary - it is more specific to the particular drug, which would modify the selection of the statin in cases of prediabetes or diabetes.

Once opinions were collected on the basis of the scientific evidence available, different recommendations have been proposed for clinical practice and offered in a panel.
Table 3: Agreements on the management of dyslipidaemia in patients with glucose metabolism alterations

\begin{tabular}{|l|l|}
\hline \multirow{4}{*}{$\begin{array}{l}\text { Screening in selected } \\
\text { patients }\end{array}$} & With diabetes or prediabetes \\
\cline { 2 - 2 } & With history of cardiovascular diseases \\
\cline { 2 - 2 } Initial therapeutic approach & With family history of cardiovascular diseases \\
\hline & With arterial hypertension \\
\hline & With abdominal obesity \\
\hline & Statin as treatment of choice \\
\hline & $\begin{array}{l}\text { Statin and dose selection according to basal } \\
\text { LDL and target LDL }\end{array}$ \\
\hline \multirow{5}{*}{$\begin{array}{l}\text { Statin replacement in case of side effects } \\
\text { control }\end{array}$} & Complete lipid profile: CT, LDL, HDL, TG \\
\hline & HbA1C in diabetics \\
\hline & Baseline glycaemia \\
\cline { 2 - 2 } & Arterial pressure \\
\hline & Hepatic enzymes \\
\hline & Renal function \\
\hline & Weight \\
\hline & Estimated cardiovascular risk \\
\hline
\end{tabular}

Table 4: Elements for drug selection in patients with glucose metabolism disorders

\begin{tabular}{|l|l|}
\hline \multirow{4}{*}{ At the time of prescribing } & LDL reduction capacity \\
\cline { 2 - 2 } & Risk of interactions \\
\hline & Complete lipid profile (CT, LDL, HDL, TG) \\
\hline & Hepatic enzymes \\
\hline & Polypharmacy \\
\hline & Estimated cardiovascular risk \\
\hline HbA1C in diabetics \\
\hline & Complete lipids profile (CT, LDL, HDL, TG) \\
\hline Hepatic enzymes \\
\hline & Estimated cardiovascular risk \\
\hline HbA1C in diabetics \\
\hline & Baseline glycaemia \\
\hline & Polypharmac \\
\hline
\end{tabular}

Table 5: Selection of statin in patients with alterations in glucose metabolism

\begin{tabular}{|c|c|}
\hline \multirow{9}{*}{ Selection based on } & Age of the patient \\
\hline & Estimated cardiovascular risk \\
\hline & Estimated diabetes mellitus risk \\
\hline & Fasting plasma glucose levels \\
\hline & Patient's HbA1c levels \\
\hline & Regular monitoring of glucose levels \\
\hline & Renal function / albuminuria levels \\
\hline & Polypharmacy, where applicable \\
\hline & $\begin{array}{l}\text { Selection of a statin that does not worsen } \\
\text { carbohydrate metabolism }\end{array}$ \\
\hline \multirow[b]{2}{*}{$\begin{array}{l}\text { General diabetogenic } \\
\text { profile }\end{array}$} & Differs among the different statins \\
\hline & $\begin{array}{l}\text { Not all have diabetogenic effects: } \\
\text { - } \quad \begin{array}{l}\text { Atorvastatin is considered the most } \\
\text { diabetogenic }\end{array} \\
\text { - Pitavastatin does not have a diabetogenic } \\
\text { profile }\end{array}$ \\
\hline Selecti & $\begin{array}{l}\text { In the opinion of the experts, pitavastatin is the } \\
\text { best choice in pre-diabetic or diabetic patients }\end{array}$ \\
\hline
\end{tabular}

\section{Recommendations for Clinical Practice}

In general, the recommendations available refer to very basic aspects, such as the special attention to high-risk patients with diabetes, the control of baseline glycaemic markers and the optimisation of the hypocholesterolemic treatment by selecting statins and doses that guarantee the achieving of the LDLc objectives with the lowest impact on 
glucose metabolism [22], even those that have shown a more favourable effect on diabetic patients with incompletely controlled diabetes mellitus type $2[23]$.

Moreover, it is necessary to consider some factors that condition the diabetogenic response of statins, pointing out the more susceptible groups. These include, in addition to individual factors, the presence of risk factors for diabetes mellitus such as BMI $>30 \mathrm{~kg} / \mathrm{m}^{2}$, triglycerides $>150 \mathrm{mg} / \mathrm{dl}$, baseline glucose $>100 \mathrm{mg} / \mathrm{dl}$, family history of diabetes mellitus, old age, post-menopausal women or the long-term use of statins $[4,9,10,22,24]$.

Therefore, and with regard to a guide that includes - and details - the main aspects to be considered in the choice of a statin for the treatment of dyslipidaemia in patients with prediabetes or diabetes mellitus, the main recommendations resulting from the opinion of the experts and the more relevant scientific evidence have been grouped in four sections, according to the variables to be considered:

- Patient characteristics:

- Assess age

- Assess individual cardiovascular risk

- Assess renal function (and albuminuria, where applicable)

○ Consider, when present:

- Multi-pathology

- Comorbidity

- Polypharmacy

- Patient's carbohydrate metabolism:

- Assess, specifically the risk of diabetes

- Consider fasting glucose levels

- Consider glycosylated haemoglobin levels

- Regularly monitor glucose levels

- Dyslipidaemia treatment:

- Consider that statins have different diabetogenic profiles, with different risks of altering carbohydrate metabolism

- Select the most convenient statin for patients with:

- High risk of diabetes (visceral obesity, metabolic syndrome)

- Prediabetes or specific circumstances

- Altered baseline glycaemia

- Oral glucose overload intolerance

- Frank diabetes mellitus

- $\quad$ Statin of choice:

- Select a statin with evidences showing that it does not worsen carbohydrate metabolism and does not increase the risk of new-onset diabetes (currently pitavastatin, according to the specifications shown in the summary of product characteristics.

- Use this statin in diabetic and pre-diabetic patients and those at risk of developing diabetes mellitus.

Finally, it is necessary to point out that other additional factors, not directly related with hypocholesterolemic potential must be considered when assessing the use of a statin in those patients with carbohydrate metabolism disorders. Among them, and with regard to a more suitable selection as indicated in the recommendations, worth mentioning in addition to the absence of diabetogenicity:
- Reduction of the residual risk of lipid origin in patients under statin treatment. This is of particular interest in patients with high cardiovascular risk (as is the case of diabetic patients or those in secondary prevention). It enables a parallel improvement in nonLDLc-dependent dyslipidaemia, based on a decrease in triglycerides and an increase in HDL-c $[25,26]$.

- Minimise the risk of pharmacological interactions through a more CYP450-independent metabolism; which permits a safer use in patients with multi-pathology, comorbidity and polypharmacy [27].

- Safety in the renal patient: This is a transcendental aspect that frequently forces an adjustment in statin dose in the presence of renal insufficiency. In this sense, recent evidences indicate that not all statins display the same renal safety profile (progression of the CKD and/or effects on albumin/proteinuria) [28]. The selection of a statin in renal patients with nephropathy whether associated with diabetes or not $[21,29]$, must guarantee its safe use. This does not seem to be always considered by prescribing practitioners [30].

In consequence, the elements to be considered to minimise a possible deterioration in glucose metabolism in patients subjected to treatment with statins are numerous although not excessively complex. Perhaps the most important thing to be aware is that, although it may be a class effect and dependent on doses, duration and intensity of the treatment, the diabetogenic potential of statins differs among them. In fact, the medicines agencies of the European member states where pitavastatin has marketing authorization, have recently recognised that the scientific evidence points that pitavastatin does not produce an increase in new cases of diabetes mellitus, being included in the respective summary of product characteristics for its consideration when prescribing statins [31]. All this must be carefully assessed especially in patients who simultaneously have a high cardiovascular disease and diabetes mellitus risk, where it is necessary to select a statin without diabetogenic effect.

Recent data [23] could be to identify a correlation between baseline serum HbAlc levels and the beneficial effects of lowering HbAlc in patients with type 2 diabetes receiving pitavastatin treatment. The correlation between baseline HbAlc and improvements in HbAlc remained significant after adjusting for age, estimated glomerular filtration rate, BMI, dose of pitavastatin and baseline HDL-C. Only the patients with poorly controlled diabetes experienced a benefit from lowering glucose with pitavastatin treatment in this study [23], and this might explain why the beneficial effect on glucose with pitavastatin treatment is not always consistent between studies [32-35]. Further large-scale trials are warranted to assess the outcomes of long-term clinical events from pitavastatin treatment; but other than decreasing LDL-C in patients with type 2 diabetes who were naive to statins (pitavastatin treatment) increasing HDL-C in patients with a lower baseline HDL-C level, and decreased $\mathrm{HbAlc}$ in those with a higher baseline $\mathrm{HbAlc}$ level.

\section{References}

1. Rius Tarruella J, Millán Núñez-Cortés J, Pedro-Botet J, Pintó Sala $X$ (2015) Statins diabetogenicity: are all the same? state of art. Clin Invest Arterioscler 27: 148-158.

2. Sattar NA, Ginsberg H, Ray K, Chapman MJ, Arca M, et al. (2014) The use of statins in people at risk of developing diabetes mellitus: evidence and guidance for clinical practice. Atheroscler Suppl 15: 1-15.

3. Coleman Cl, Reinhart K, Kluger J, White CM (2008) The effect of statins on the development of new-onset type 2 diabetes: a metaanalysis of randomized controlled trials. Curr Med Res Opin 24: 1359-1362. 
4. Mora S, Glynn RJ, Hsia J, MacFadyen JG, Genest J, et al. (2010) Statins for the primary prevention of cardiovascular events in women with elevated high-sensitivity C-reactive protein or dyslipidemia: results from the Justification for the Use of Statins in Prevention: An Intervention Trial Evaluating Rosuvastatin (JUPITER) and metaanalysis of women from primary prevention trials. Circulation 121: 1069-1077.

5. Rajpathak SN, Kumbhani DJ, Crandall J, Barzilai N, Alderman M, et al. (2009) Statin therapy and risk of developing type 2 diabetes: a meta-analysis. Diabetes Care 32: 1924-1929.

6. Freeman DJ, Norrie J, Sattar N, Neely RD, Cobbe SM, et al. (2001) Pravastatin and the development of diabetes mellitus: evidence for a protective treatment effect in the West of Scotland Coronary Prevention Study. Circulation 103: 357-362.

7. The Long-Term Intervention with Pravastatin in Ischaemic Disease (LIPID) Study Group (1998) Prevention of Cardiovascular Events and Death with Pravastatin in Patients with Coronary Heart Disease and a Broad Range of Initial Cholesterol Levels. N Engl J Med 339: 13491357.

8. Shepherd J, Blauw GJ, Murphy MB, Bollen EL, Buckley BM, et al. (2002) Pravastatin in elderly individuals at risk of vascular disease (PROSPER): a randomised controlled trial. Lancet 360: 1623-1630.

9. Sattar N, Preiss D, Murray HM, Welsh P, Buckley BM, et al. (2010) Statins and risk of incident diabetes: a collaborative meta-analysis of randomised statin trials. Lancet 375: 735-742.

10. Zaharan NL, Williams D, Bennett K (2013) Statins and risk of treated incident diabetes in a primary care population. $\mathrm{Br} \mathrm{J}$ Clin Pharmacol 75: $1118-1124$

11. Carter AA, Gomes T, Camacho X, Juurlink DN, Shah BR, et al. (2013) Risk of incident diabetes among patients treated with statins: population based study. BMJ 346: f2610.

12. Preiss D, Seshasai SR, Welsh P, Murphy SA, Ho JE, et al. (2011) Risk of incident diabetes with intensive dose compared with moderatedose statin therapy: a meta-analysis. JAMA 305: 2556-2564.

13. Navarese EP, Buffon A, Andreotti F, Kozinski $M$, Welton $N$, et al. (2013) Meta-analysis of impact of different types and doses of statins on new-onset diabetes mellitus. Am J Cardiol 111: 1123-1130.

14. Vallejo-Vaz AJ, Kondapally Seshasai SR, Kurogi K, Michishita I, Nozue T, et al. (2015) Effect of pitavastatin on glucose, HbA1c and incidence diabetes: A meta-analysis of randomized controlled clinical trials in individuals without diabetes. Atherosclerosis 241: 409-418.

15. Yamazaki T, Kishimoto J, Ito C, Noda M, Odawara M, et al. (2011) Japan prevention trial of diabetes by pitavastatin in patients with impaired glucose tolerance (The J-PREDICT study): rationale, study design, and clinical characteristics of 1269 patients. Diabetol Int 2: 134-140.

16. Barrios V, Escobar C (2016) Clinical benefits of pitavastatin: focus on patients with diabetes or at risk of developing diabetes. Future Cardiol 12: $449-466$

17. Pedro Botet J, Barrios V, Pascual V, Ascaso JF, Cases A, et al. (2016) Delphi consensus on management of dyslipidaemia in patients with impaired glucose metabolism: Diana study. Clin Investig Arterioscler 28: $132-140$.

18. Millán Núñez-Cortés J, Cases Amenós A, Ascaso Gimilio JF, Barrios Alonso V, Pascual Fuster V, et al. (2016) Consensus on the statin of choice in patients with impaired glucose metabolism: Results of the DIANA study. Am J Cardiovasc Drugs 17: 135-142.
19. Serrano A, Pascual V (2016) Opinion from physicians on the need for dyslipidemia screening in cardiovascular risk. Similarities and differences between primary care and other specialties. The DIANA study. SEMERGEN.

20. Dalkey NC (1969) The Delphi method: an experimental study of group opinion. Santa Monica: Rand Corporation.

21. Kimura K, Shimano H, Yokote K, Urashima M, Teramoto T, et al. (2010) Effects of pitavastatin (LIVALO Tablet) on the estimated glomerular filtration rate (EGFR) in hypercholesterolemic patients with chronic kidney disease. Subanalysis of the LIVALO Effectiveness and Safety (LIVES) Study. J Atherosclr Thromb 17: 601-609.

22. Backes JM, Kostoff MD, Gibson CA, Ruisinger JF (2016) Statinassociated diabetes mellitus: review and clinical guide. South Med J 109: $167-173$

23. Huang $\mathrm{CH}$, Huang YY, Hsu BR (2016) Pitavastatin improves glycated hemoglobin in patients with poorly controlled type 2 diabetes. J Diabetes Investig 7: 769-776.

24. Culver AL, Ockene IS, Balasubramanian R, Olendzki BC, Sepavich DM, et al. (2012) Statin use and risk of diabetes mellitus in postmenopausal women in the Women's Health Initiative. Arch Intern Med 172: 144-152.

25. Stender S, Budinski D, Gosho M, Hounslow N (2013) Pitavastatin shows greater lipid-lowering efficacy over 12 weeks than pravastatin in elderly patients with primary hypercholesterolaemia or combined (mixed) dyslipdaemia. Eur J Prev Cardiol 20: 40-53.

26. Teramoto T, Shimano H, Yokote K, Urashima M (2009) Effects of pitavastatin (LIVALO Tablet) on high density lipoprotein cholesterol (HDL-C) in hypercholesterolemia. J Atheroscler Thromb 16: 654-661.

27. Catapano AL (2010) Pitavastatin-pharmacological profile from early phase studies. Atheroscler Suppl 11: 3-7.

28. Su X, Zhang L, Lv J, Wang J, Hou W, et al. (2016) Effect of Statins on Kidney Disease Outcomes: A Systematic Review and Meta-analysis. Am J Kidney Dis 67: 881-892.

29. Hanai K, Babazono T, Takemura S, Toyonaga A, Yoshida N, et al (2015) Comparative effects of statins on the kidney function in patients with type 2 diabetes. J Atheroscler Thromb 22: 618-627.

30. Cases Amenós A, Pedro-Botet Montoya J, Pascual Fuster V, Barrios Alonso V, Pintó Sala X, et al. (2016) Delphi Consensus on the Diagnosis and Management of Dyslipidemia in Patients With Chronic Renal Disease: Post-Hoc Analysis of the DIANA Study. Nefrologia 36: 679-686.

31. Summary of product characteristics (2016) Authorisation date in European countries, member state of reference, United Kingdom.

32. Daido H, Horikawa Y, Takeda J (2014) The effects of pitavastatin on glucose metabolism in patients with type 2 diabetes with hypercholesterolemia. Diabetes Res Clin Pract; 106: 531-537.

33. Cho Y, Choe E, Lee YH, Seo JW, Choi Y, et al. (2014) Risk of diabetes in patients treated with HMG-CoA reductase inhibitors. Metabolism 64: $482-488$

34. Gumprecht J, Gosho M, Budinski D, Hounslow N (2011) Comparative long-term efficacy and tolerability of pitavastatin $4 \mathrm{mg}$ and atorvastatin 20-40 mg in patients with type 2 diabetes mellitus and combined (mixed) dyslipidaemia. Diabetes Obes Metab 13: 1047-1055.

35. Mita T, Nakayama S, Abe H, Gosho M, lida H, et al. (2013) Comparison of effects of pitavastatin and atorvastatin on glucose metabolism in type 2 diabetic patients with hypercholesterolemia. J Diabetes Investig 4: 297-303. 